\title{
The Linear Algebra for Linked Loci with Mutation
}

\section{IVAR HEUCH*}

Department of Human Genetics, University of Michigan, Ann Arbor, Michigan

Communicated by Samuel Karlin

\section{ABSTRACT}

A linear algebra is defined describing this situation: Any number of loci are linked with arbitrary linkage distribution, and each allele may mutate into the other alleles with given frequencies. Mutations at different loci are assumed independent. It is shown that this gives a genetic algebra (in the sense of Gonshor or Schafer) which is also a special train algebra, and the train roots are found.

\section{INTRODUCTION}

Linear commutative nonassociative algebras over the complex numbers were introduced in mathematical genetics by Etherington $[4,6]$. The fundamental idea is to define a basis $G_{1}, G_{2}, \ldots, G_{n}$ with one-to-one correspondence to the genotypes $g_{1}, g_{2}, \ldots, g_{n}$ considered, and then give a multiplication table so that the product $G_{i} G_{j}$ of two basis elements will be equal to a linear combination $\sum_{k} p_{i j k} G_{k}$, where $p_{i j k}$ is the probability of getting genotype $g_{k}$ in a cross between $g_{i}$ and $g_{j}$ individuals. The genotype distribution after random mating between two infinite populations may be found by calculating the product of two suitable linear combinations of the basis elements. Products with more than two factors will give the offspring distribution in more complicated pedigrees.

One of the reasons for considering such algebras is the possibility of proving general theorems for different kinds of sequences of algebra elements, representing successive generations in various mating systems, as for instance Theorems 2.1 and 2.2 in Ref. 8, Theorem 3 in Ref. 12 and Theorem 5.1 in Ref. 11. Most such theorems apply to what is defined by Gonshor [9] to be genetic algebras: An algebra is genetic if it is possible to find a basis $C_{0}, C_{1}, \ldots, C_{n}$ giving a multiplication table

$$
C_{i} C_{j}=\sum_{k=0}^{n} \gamma_{i j k} C_{k},
$$

where $\gamma_{000}=1, \gamma_{0 j k}=0$ for $k<j$ and $\gamma_{i j k}=0$ for $k \leqslant \max (i, j)$; $i, j=1,2, \ldots, n$. This basis is said to be canonical. The train roots 1 ,

\footnotetext{
* Present address: Institute of General Genetics, University of Oslo, Oslo, Norway.
}

(C) American Elsevier Publishing Company, Inc., 1973 
$\gamma_{011}, \ldots, \gamma_{0_{n n}}$ are important when we want to construct expressions for the general member of various sequences. The definition of a genetic algebra given by Schafer [15] is equivalent to the one above (Theorem 2.1 in Ref. [9]), but Gonshor's is more suitable for practical applications. An algebra is baric if there exists a nontrivial homomorphism $w$ to the scalar field [4]. Then $w$ is called the weight function. Genetic algebras are baric with $w\left(C_{0}\right)=1, w\left(C_{j}\right)=0$ for $j>0$. A special train algebra $[4,5]$ is a baric algebra where the nilidieal $N$ of elements with weight 0 is nilpotent, and where all powers of $N$ are ideals in the total algebra. Every special train algebra is genetic [5]; an example of a genetic algebra which is not a special train algebra is furnished by the copular algebra for simple Mendelian inheritance treated in Ref. 15.

The deterministic model for any number of arbitrarily linked loci was first studied by Geiringer [7], and later by Bennett [1]. Limit theorems for a more general situation are given by Ellison [2]. A treatment by means of linear algebras is found in [13]. Algebras for linked loci are also used in [14] and [10], though the methods applied are quite different from ours. Algebras for mutation have been considered in connection with one autosomal locus and sex-linkage [8], and polyploidy [8,9].

\section{ELEMENTARY ALGEBRAS}

Assume that $E$ is an algebra with basis $A_{0}, A_{1}, \ldots, A_{r}$ and multiplication table

$$
A_{i} A_{j}=\left(A_{i}+A_{j}\right) / 2 ; \quad i, j=0,1, \ldots, r .
$$

Then $E$ is called elementary ([13], Sec. 2), and we will refer to the basis used here as the natural basis. $E$ is baric with weight given by $w\left(A_{i}\right)=1$ for all $i$. Using any basis $C_{0}, C_{1}, \ldots, C_{\mathrm{r}}$ with $w\left(C_{0}\right)=1, w\left(C_{i}\right)=0$ for $i>0$, we find a multiplication table

$C_{0}^{2}=C_{0} ; \quad C_{0} C_{i}=C_{i} / 2, \quad i=1,2, \ldots, r ; \quad C_{i} C_{j}=0$ otherwise. (Introduce for instance $C_{0}=A_{0}, C_{i}=A_{0}-A_{i}$.) Hence the elementary algebra $E$ is a special train algebra with train roots 1 and $\frac{1}{2}$, the latter with multiplicity $r$, see Ref. 13 .

Let $E_{1}, E_{2}, \ldots, E_{k}$ be $k$ elementary algebras such that $E_{m}$ has natural basis $A_{m 0}, A_{m 1}, \ldots, A_{m r_{m}}$. We now introduce a vector space $V_{k}$ with basis elements $A_{1 i_{1}} A_{2 i_{2}} \cdots A_{k i_{k}}$ constructed by juxtaposition of basis elements from the $k$ algebras. Following Holgate ([13], Sec. 3) we will write

$$
\prod_{m=1}^{k}\left(\sum_{j=0}^{r_{m}} a_{m j} A_{m j}\right)
$$

for the element

$$
\sum_{j_{1}} \cdots \sum_{j_{k}} a_{1 j_{1}} a_{2 j_{2}} \cdots a_{k j_{k}} A_{1 j_{1}} A_{2 j_{2}} \cdots A_{k j_{k}},
$$


in $V_{k}$. Suppose that for $m=1,2, \ldots, k, B_{m 0}, B_{m 1}, \ldots, B_{m r_{m}}$ is an arbitrary basis in $E_{m}$. Then it is seen that any sequence of all elements of the form $B_{1 j_{1}} B_{2 j_{2}} \cdots B_{k j_{k}}$ will give a basis in $V_{k}$.

The linked product $E^{*}$ of the elementary algebras $E_{1}, E_{2}, \ldots, E_{k}$ was introduced by Holgate in Ref. 13. This is an algebra over $V_{k}$ with multiplication given by

$$
\begin{aligned}
& \left(A_{1 i_{1}} A_{2 i_{2}} \cdots A_{k i_{k}}\right)\left(A_{1 j_{1}} A_{2 j_{2}} \cdots A_{k j_{k}}\right) \\
& \quad=\left(A_{1 i_{1}} A_{2 i_{2}} \cdots A_{k i_{k}}+A_{1 j_{1}} A_{2 j_{2}} \cdots A_{k j_{k}}\right) / 2 .
\end{aligned}
$$

$E^{*}$ will itself be elementary with the sequence of all $A_{1 i_{1}} A_{2 i_{2}} \cdots A_{k i_{k}}$ as the natural basis. When $C_{m 0}, C_{m 1}, \ldots, C_{m r_{m}}$ for $m=1,2, \ldots, k$ are arbitrary canonical bases for the original algebras, then we find that $C_{10} C_{20} \cdots C_{k 0}$ will have weight 1 in $E^{*}$, and all other $C_{1 i_{1}} C_{2 i_{2}} \cdots C_{k i_{k}}$ have weight 0 . Thus any sequence of all $C_{1 i_{1}} C_{2 i_{2}} \cdots C_{k i_{k}}$ with $C_{10} C_{20} \cdots$ $C_{k 0}$ as the first member will constitute a canonical basis in $E^{*}$.

\section{RECOMBINATION ALGEBRAS}

We set $S=\{1,2, \ldots, k\}$. Suppose that $U^{\prime}$ and $U^{\prime \prime}$ are complementary subsets of $S$. Then we let

$$
U=\left(U^{\prime}, U^{\prime \prime}\right)=\left(U^{\prime \prime}, U^{\prime}\right),
$$

stand for the (non-ordered) partition of $S$ given by $U^{\prime}$ and $U^{\prime \prime}$. The collection of all such partitions will be designated as $W(S)$. This notation is identical with that introduced in Ref. 14. For given elementary algebras $E_{1}, \ldots, E_{k}$ we will now define the recombination algebra $X(U)$ with respect to $U$ formed from $E_{1}, \ldots, E_{k}$. This is to be an algebra over $V_{k}$ with multiplication given by

$$
\begin{aligned}
& \left(\prod A_{m i_{m}}\right)\left(\prod A_{m j_{m}}\right) \\
& =\left[\left(\prod_{m \in U^{\prime}} A_{m i_{m}}\right)\left(\prod_{m \in U^{\prime \prime}} A_{m j_{m}}\right)+\left(\prod_{m \in U^{\prime \prime}} A_{m i_{m}}\right)\left(\prod_{m \in U^{\prime}} A_{m j_{m}}\right)\right] / 2,
\end{aligned}
$$

where the $A_{m i}$ are elements in the natural bases as before. We adopt the convention that products without specifications should be taken over all $m \in S$. If $U^{\prime}=S$ and $U^{\prime \prime}=\phi$, then $X(U)=E^{*}$.

Consider in particular $X(U)$ for $k=2$ and $U=(1,2)$. Let $B$ and $B^{\prime}$ be elements in $E_{1}$ with weight 1 , and $C$ and $C^{\prime}$ elements in $E_{2}$ also having weight 1 . Expressing these elements in terms of the natural bases in $E_{1}$ and $E_{2}$, we find that

$$
(B C)\left(B^{\prime} C^{\prime}\right)=\left(B C^{\prime}+B^{\prime} C\right) / 2 .
$$

Now let $D_{10}, D_{11}, \ldots, D_{1 r_{1}}$ and $D_{20}, D_{21}, \ldots, D_{2 r_{2}}$ be any canonical bases for $E_{1}$ and $E_{2}$, respectively. Then all $D_{10}-D_{1 i_{1}}$ and $D_{20}-D_{2 i_{2}}$ have weight 1 in $E_{1}$ and $E_{2}$, and applying Eq. (2) we construct the following multiplication table in $X(U)$ : 


$$
\begin{array}{cc}
\left(D_{10} D_{20}\right)^{2}=D_{10} D_{20}, & \left(D_{10} D_{20}\right)\left(D_{10} D_{2 j}\right)=D_{10} D_{2 j} / 2, \\
\left(D_{10} D_{20}\right)\left(D_{1 i} D_{20}\right)=\left(D_{1 i} D_{20}\right) / 2, & \left(D_{1 i} D_{20}\right)\left(D_{10} D_{2 j}\right)=D_{1 i} D_{2 j} / 2,(3) \\
i=1,2, \ldots, r_{1}, & j=1,2, \ldots, r_{2},
\end{array}
$$

all other products $\left(D_{1 i} D_{2 j}\right)\left(D_{1 h} D_{2 s}\right)$ being equal to 0 .

We return to the case with arbitrary $k$ and $U$. If we denote the linked product of all $E_{m}$ with $m \in U^{\prime}$ by $L_{1}$, and similarly the linked product of $E_{m}$ with $m \in U^{\prime \prime}$ by $L_{2}$, then $L_{1}$ and $L_{2}$ will be elementary. Furthermore, the recombination algebra $X(U)$ defined by Eq. (1) will be identical with the recombination algebra formed from $L_{1}$ and $L_{2}$ with respect to the partition $U=(1,2)$. Assume as before that $C_{m 0}, C_{m 1}, \ldots, C_{m r_{m}}$ is any canonical basis for $E_{m}$. Then $L_{1}$ has a canonical basis consisting of the elements $\prod_{m \in V^{\prime}} C_{m i m}$ obtained when all $i_{m}$ vary between 0 and $r_{m}$. The first element in this basis must be $\prod_{m \in U^{\prime}} C_{m 0}$, but apart from this the ordering is arbitrary. We now want to construct the general multiplication table in $X(U)$ using the table (3). For this purpose we set $\prod_{m \in U^{\prime}} C_{m 0}=D_{10}$, and then each of the elements $\prod_{m \in U^{\prime}} C_{m i_{m}}$ with at least one $i_{m} \neq 0$ is identified with one of $D_{11}, D_{12}, \ldots$ used in Eq. (3). ( $r_{1}$ in the basis in Eq. (3) will correspond to $\prod_{m \in U^{\prime}}\left(r_{m}+1\right)-1$.) $L_{2}$ may be treated in the same way, and so we find in $X(U)$

$$
\left(\prod C_{m 0}\right)^{2}=\left(D_{10} D_{20}\right)^{2}=\prod C_{m 0} .
$$

If there exists a $m \in U^{\prime}$ with $i_{m} \neq 0$ we have (with a suitable $t \neq 0$ )

$$
\begin{aligned}
& \left(\prod C_{m 0}\right)\left(\prod_{m \in U^{\prime}} C_{m i_{m}} \prod_{m \in U^{\prime \prime}} C_{m 0}\right) \\
& \quad=\left(D_{10} D_{20}\right)\left(D_{12} D_{20}\right)=1 / 2\left(\prod_{m \in U^{\prime}} C_{m i_{m}} \prod_{m \in U^{\prime \prime}} C_{m 0}\right) .
\end{aligned}
$$

This result is symmetric in $U^{\prime}$ and $U^{\prime \prime}$. Moreover,

$$
\left(\prod C_{m 0}\right)\left(\prod_{m \in U^{\prime}} C_{m i_{m}} \prod_{m \in U^{\prime \prime}} C_{m j_{m}}\right)=\left(D_{10} D_{20}\right)\left(D_{1 i} D_{2 u}\right)=0
$$

if $i_{m} \neq 0$ for at least one $m \in U^{\prime}$ and $j_{n} \neq 0$ for one $n \in U^{\prime \prime}$. Multiplying two elements $\prod C_{m i_{m}}$ and $\prod C_{m j_{m}}$ with $i_{m} \neq 0$ for some $m$ and $j_{n} \neq 0$ for some $n$, we see that the product is 0 if any of $U^{\prime}$ or $U^{\prime \prime}$ contains an $m$ with $i_{m} \neq 0$ and at the same time an $n$ with $j_{n} \neq 0$. Otherwise we have, with suitable $t$ and $u$,

$$
\left(\prod C_{m i_{m}}\right)\left(\prod C_{m j_{n}}\right)=\left(D_{1 t} D_{20}\right)\left(D_{10} D_{2 u}\right)=1 / 2\left(D_{1 i} D_{2 u}\right) \text {. }
$$

Here $D_{1 t} D_{2 u}$ will correspond to a $\prod C_{m v_{m}}$, where $v_{m}=i_{m}$ if $i_{m} \neq 0$, $v_{m}=j_{m}$ if $j_{m} \neq 0$, and $v_{m}=0$ if $i_{m}=j_{m}=0$.

\section{THE BIOLOGICAL SITUATION}

We consider haploid individuals with $k$ linked autosomal loci without selection. The possible alleles in locus number $m$ are $A_{m 0}, A_{m 1}, \ldots, A_{m r_{m}}$. What happens regarding recombination during mciosis in a particular 
zygote may be described by specifying a partition $U=\left(U^{\prime}, U^{\prime \prime}\right)$ of the set $S$. Genes in loci with indices in the same set $U^{\prime}$ or $U^{\prime \prime}$ will behave as an entity, while the blocks represented by $U^{\prime}$ and $U^{\prime \prime}$ are recombined. The degree of linkage hetween the $k$ loci is given by a linkage distribution $\{\lambda(U)\}$ over $W(S)$. For this distribution we use the same notation as Reiersøl in Ref. 14.

The allele $A_{m i}$ is assumed to mutate to $A_{m j}$ with a frequency of $v_{m i j}$ per generation, and it will remain unchanged with a frequency $v_{m i i}(i, j=$ $\left.0,1, \ldots, r_{m} ; i \neq j\right)$. For each locus $m$ we thus obtain a $\left(r_{m}+1\right) \times\left(r_{m}+1\right)$ mutation matrix $\mathbf{v}_{m}=\left(v_{m i j}\right)_{i, j}$, which may also be regarded as the transition matrix for a discrete time Markov chain. We assume that it is possible to find a complete set linearly independent eigenvectors for every transition matrix. It is also assumed that genes in different loci mutate independently.

Consider the Markov chain for locus number $m$. Let the ordering of the states $0,1, \ldots, r_{m}$ in the chain be such that all states in the same recurrent class follow each other, and all recurrent states precede the transient ones. The recurrent classes may then be given as

$$
\left\{0,1, \ldots, y_{0}\right\},\left\{y_{0}+1, \ldots, y_{1}\right\}, \ldots,\left\{y_{s_{m}-1}+1, \ldots, y_{s_{m}}\right\} .
$$

Thus the eigenvalue $\mu=1$ has multiplicity $s_{m}+1$. Let the stationary distribution for recurrent class number $t$ be given by the quantities $p_{j} ; j=y_{t-1}+1, \ldots, y_{t}$ (with an obvious modification for $t=0$ ). We may then take as a left eigenvector for $\mu=1$ a row vector consisting of zeros except for elements $j=y_{t-1}+1, \ldots, y_{t}$, where the values $p_{j}$ are inserted. The remaining $r_{m}-s_{m}$ left eigenvectors, corresponding to eigenvalues different from 1 , will be written as $\left(x_{t 0}, x_{t 1}, \ldots, x_{t r_{m}}\right)$; $t=s_{m}+1, s_{m}+2, \ldots, r_{m}$.

\section{THE COMPLETE ALGEBRA}

Without mutations the inheritance for each separate locus would be described by an elementary algebra $E_{m}$ with natural basis $A_{m 0}, A_{m 1}, \ldots$, $A_{m r_{m}}$ (we identify the basis elements with the genotypes). The effect of mutation during one generation is obtained by applying the linear operator $M$ defined by

$$
M\left(A_{m i}\right)=\sum_{j=0}^{r_{m}} v_{m i j} A_{m j}
$$

We now construct a particular canonical basis for each $E_{m}$. As the first element we take

$$
C_{m 0}=\sum_{j=0}^{y_{0}} p_{j} A_{m j}
$$


which has weight 1 . For $i=1,2, \ldots, s_{m}$ we set

$$
C_{m i}=C_{m 0}-\sum_{j=y_{i-1}+1}^{y_{i}} p_{j} A_{m j}
$$

with wcight 0 , and for $i=s_{m}+1, s_{m}+2, \ldots, r_{m}$ we define

$$
C_{m i}=\sum_{j=0}^{r_{m}} x_{i j} A_{m j}
$$

Since the right eigenvector $(1,1, \ldots, 1)^{\prime}$ for $\mu=1$ and each $\left(x_{i 0}, x_{11}, \ldots\right.$, $x_{i r_{m}}$ ) are orthogonal, these $C_{m i}$ will also have weight 0 in $E_{m}$. Because of the existence of a complete set of eigenvectors, $C_{m 0}, C_{m 1}, \ldots, C_{m r_{m}}$ will actually constitute a basis, and it must be canonical since $E_{m}$ is elementary. Furthermore,

$$
M\left(C_{m 0}\right)=C_{m 0} ; \quad M\left(C_{m i}\right)=C_{m i}, \quad i=1,2, \ldots, s_{m},
$$

and, if $\mu_{m i}$ is the eigenvalue corresponding to $\left(x_{i 0}, \ldots, x_{i r_{m}}\right)$, then

$$
M\left(C_{m i}\right)=\mu_{m i} C_{m i}, \quad i=s_{m}+1, \ldots, r_{m} .
$$

The eigenvalues $\mu_{m 0}, \mu_{m 1}, \ldots, \mu_{m s_{m}}$ are equal to 1 , hence Eq. (11) may be used for all $i=0,1, \ldots, r_{m}$.

The simultaneous inheritance for all $k$ loci without mutation may be described by an algebra $X$ over $V_{k}$ given by

$$
\begin{aligned}
& \left(\prod A_{m i_{m}}\right)\left(\prod A_{m j_{m}}\right) \\
& \quad=1 / 2 \sum_{U \in W(S)} \lambda(U)\left(\prod_{m \in U^{\prime}} A_{m i_{m}} \prod_{m \in U^{\prime \prime}} A_{m j_{m}}+\prod_{m \in U^{\prime \prime}} A_{m i_{m}} \prod_{m \in U^{\prime}} A_{m j_{m}}\right) .
\end{aligned}
$$

It is seen that $X$ may be expressed as a mixture (see Ref. 13) of the recombination algebras $X(U)$

$$
X=\sum_{U \in W(S)} \lambda(U) X(U)
$$

Introducing the canonical bases constructed above for $E_{1}, E_{2}, \ldots, E_{k}$ and using Eqs. (4)-(7), we can now find the multiplication table for $X$ in terms of the basis of all $C_{1 i_{1}} C_{2 i_{2}} \cdots C_{k i_{k}}$. We then obtain the multiplication rules in the complete algebra $X_{M}$ for the case with mutations by operating with $M$ on the right hand side of the equations in this table and using

$$
M\left(C_{1 i_{1}} C_{2 i_{2}} \cdots C_{k i_{k}}\right)=M\left(C_{1 i_{1}}\right) M\left(C_{2 i_{2}}\right) \cdots M\left(C_{k i_{k}}\right),
$$

which is valid because of the independence for mutations in different loci.

For any $k$-tuple $i=\left(i_{1}, i_{2}, \ldots, i_{k}\right)$ of non-negative integers we will define a subset $Q(i)=Q\left(i_{1}, \ldots, i_{k}\right)$ in $W(S)$. This subset is to consist of those partitions $U=\left(U^{\prime}, U^{\prime \prime}\right)$ of $S$ where all $m$ with nonzero $i_{m}$ are elements in the same set $U^{\prime}$ or $U^{\prime \prime}$. We now find in $X_{M}$

With at least one $i_{m} \neq 0$ we have

$$
\left(\prod C_{m 0}\right)^{2}=\prod C_{m 0} \text {. }
$$

$$
\left(\prod C_{m 0}\right)\left(\prod C_{m_{m}}\right)=\left(1 / 2 \sum_{v \in Q(i)} \lambda(U) \cdot \prod \mu_{m i_{m}}\right) \prod C_{m i_{m}}
$$


Assume that $i=\left(i_{1}, \ldots, i_{k}\right)$ and $j=\left(j_{1}, \ldots, j_{k}\right)$ satisfy the condition that if $i_{m} \neq 0$ and $j_{n} \neq 0$ then $m \neq n$, and that $i_{m} \neq 0$ and $j_{n} \neq 0$ for at least one $m$ and $n$. Then

$$
\left(\prod C_{m i_{m}}\right)\left(\prod C_{m j_{m}}\right)=\left(1 / 2 \sum_{U \in R(i, j)} \lambda(U) \cdot \prod\left[\mu_{m t_{m}}\right) \prod C_{m t_{m}}\right. \text {. }
$$

Here $t_{1}, \ldots, t_{k}$ are given by $t_{m}=i_{m}$ if $i_{m} \neq 0, t_{m}=j_{m}$ if $j_{m} \neq 0, t_{m}=0$ otherwise, and $R(i, j)$ is the collection of the partitions $U$ where $m$ with $i_{m} \neq 0$ are contained in one of the sets $U^{\prime}$ and $U^{\prime \prime}$ and $n$ with $j_{n} \neq 0$ are contained in the other. If there exists an $m$ with both $i_{m} \neq 0$ and $j_{m} \neq 0$, then

$$
\left(\prod C_{m i_{m}}\right)\left(\prod C_{m j_{m}}\right)=0
$$

Thus we have established:

THEOREM 1

$X_{M}$ is a genetic algebra. The train roots are 1 and all values

$$
1 / 2 \sum_{v \in Q(i)} \lambda(U) \cdot \prod \mu_{m i_{m}}
$$

obtained when $i=\left(i_{1}, \ldots, i_{k}\right)$ varies under the condition that at least one $i_{m}$ is nonzero. A canonical basis can consist of all $C_{1 i_{1}} C_{2 i_{2}} \cdots C_{k_{i_{k}}}$ (where the $C_{m i}$ are defined by Eqs. (8)-(10)); the ordering in this basis may for instance be lexicographic, that is $C_{1 i_{1}} \cdots C_{k i_{k}}$ precedes $C_{1 j_{1}} \cdots C_{k j_{k}}$ if $i_{1}=j_{1}, \ldots, i_{u-1}=j_{u-1}, i_{u}<j_{u}$ for some $u$.

We also have

\section{THEOREM 2}

$X_{M}$ is a special train algebra.

Proof. The subspace $N$ of all elements in $X_{M}$ of weight 0 is spanned by the elements

$$
C_{1 i_{1}} C_{2 i_{2}} \cdots C_{k i_{k}}
$$

with at least one $i_{m} \neq 0$. Let $T_{d}$ be the collection of all elements (17) equal to a product of $d$ other elements (17) where the shape of the product is primary (that is, where the factors are absorbed one at a time, see Ref. 3, Sec. 2). Suppose that $P$ is an arbitrary primary product with $d$ factors in $N$. Expressing these factors as linear combinations of elements (17), we see that the total product $P$ must be a linear combination of elements in $T_{d}$, since it follows from Eqs. (14) and (15) that every product

$$
\left(C_{1 i_{1}} \cdots C_{k i_{k}}\right)\left(C_{1 j_{1}} \cdots C_{k j_{k}}\right)
$$

in $N$ will either be 0 or may be expressed by one single element (17). The power $N^{d}$ of $N$ is the set of all linear combinations of such products $P$, hence $N^{d}$ will be the space spanned by $T_{d}$. Then, according to Eq. (13), every product $\left(C_{10} C_{20} \cdots C_{k 0}\right) F$ with $F \in N^{d}$ will lie in $N^{d}$, and $N^{d}$ will 
be an ideal in $X_{M}$. This is sufficient for concluding that $X_{M}$ is a special train algebra, since it is already established that $X_{M}$ is genetic.

The actual extent of $T_{d}$ and $N^{d}$ may vary with the choice of values $v_{m i j}$ and $\lambda(U)$. If $\lambda(U)>0$ for all $U \in W(S)$, and no eigenvalues $\mu_{m i}$ are 0 , then $T_{d}$ will consist of those $C_{1 i_{1}} C_{2 i_{2}} \cdots C_{k i_{k}}$ with at least $d$ nonzero $i_{m}$. This may be shown by induction with respect to $d$. On the other hand, consider as an example the case with $k=4$ without mutation where $\lambda(i j, t u)>0$, all $\lambda(i j t, u)=0$ and $\lambda(1234)>0$. Then $T_{2}$ will consist of all $C_{1 i_{1}} C_{2 i_{2}} C_{3 i_{3}} C_{4 i_{4}}$ with at least two nonzero $i_{m}, T_{3}$ of all such elements with three or four nonzero $i_{m}$, while $T_{4}$ is empty and $N^{4}=\{0\}$.

\section{CONNECTIONS WITH PREVIOUS RESULTS}

The case without mutations corresponds to $v_{m i i}=1, v_{m i j}=0$ for $i \neq j$. Then the definitions (8)-(10) reduce to $C_{m 0}=A_{m 0}, C_{m i}=A_{m 0}-A_{m i}$, and our theorems may be compared with the results in Ref. 13, Sec. 5 . It is there stated that a proof has been given for the proposition that $X$ is a special train algebra. But the definition quoted of this concept in Ref. 13, Sec. 1 is identical with Gonshor's later definition [9] of a genetic algebra rather than Etherington's definition of a special train algebra. Thus the conclusion in Ref. 13 gives the same as our Theorem 1, but Theorem 2 is new even for the particular case with no mutations. (However, the situation with only 3 loci was treated in Ref. 4.) When all $\mu_{m i}=1$ the train roots (16) are seen to coincide with the values given in Eq. (24) in Ref. 13.

The treatment in Ref. 13 is based on a different mixture than Eq. (12), and the algebraic manipulations seem more complicated. One of the reasons for this may be that the scalars $\alpha(I)$ appearing in the mixture in Ref. 13 do not correspond to any obvious parameters connected with the linkage distribution characterizing the biological situation, although this situation is defined in Ref. 13 in a manner similar to ours, with a quantity $p(I)$ corresponding to our $\lambda(U)$. Furthermore, the algebras $A(I)$ in Ref. 13 are introduced by consideration of blocks of loci with successive indices, even though linearity of chromosomes is not used in the deductions, and the model could be applied even in theoretical situations where the linkage distribution would contradict such a hypothesis.

The consequences of Theorems 1 and 2 for various sequences and for the diploid case will be treated elsewhere.

\section{REFERENCES}

1 J. H. Bennett, On the theory of random mating, Ann. Eugenics 18, 311 (1954).

2 B. E. Ellison, Limit theorems for random mating in infinite populations, J. Appl. Prob. 3, 94 (1966). 
3 I. M. H. Etherington, On nonassociative combinations, Proc. Roy. Soc. Edinburgh 59, 153 (1939).

4 I. M. H. Etherington, Genetic algebras, Proc. Roy. Soc. Edinburgh 59, 242 (1939).

5 I. M. H. Etherington, Special train algebras, Quart.J. Math. (Oxford) (2) 12, 1 (1941),

6 I. M. H. Etherington, Nonassociative algebra and the symbolism of genetics, Proc, Roy. Soc. Edinburgh B 61, 24 (1941).

$7 \mathrm{H}$. Geiringer, On the probability theory of linkage in Mendelian heredity, Ann. Math. Statist. 15, 25 (1944).

$8 \mathrm{H}$. Gonshor, Special train algebras arising in genetics, Proc. Edinburgh Math. Soc, (2) 12, 41 (1960).

9 H. Gonshor, Contributions to genetic algebras, Proc. Edinburgh Math. Soc. (2) 17, 289 (1971).

$10 \mathrm{I}$. Heuch, $k$ loci linked to a sex factor in haploid individuals, Biom. Zeitschrift, 13, 57 (1972).

$11 \mathrm{I}$. Heuch, Sequences in genetic algebras for overlapping generations, Proc. Edinburgh Math. Soc. (to be published).

12 P. Holgate, Sequences of powers in genetic algebras, J. London Math. Soc. 42, 489 (1967).

13 P. Holgate, The genetic algebra of $k$ linked loci, Proc. London Math. Soc. (3) 18, 315 (1968).

140 . Reiersøl, Genetic algebras studied recursively and by means of differential operators, Math. Scand. 10, 25 (1962).

15 R. D. Schafer, Structure of genetic algebras, Amer. J. Math. 71, 121 (1949).

Received April, 1972 